\title{
Investigation of speckle pattern dynamics by laser speckle contrast imaging
}

\section{Anton Sdobnov, Alexander Bykov, Alexey Popov, Evgeny Zherebtsov, Igor Meglinski}

Anton Sdobnov, Alexander Bykov, Alexey Popov, Evgeny Zherebtsov, Igor Meglinski, "Investigation of speckle pattern dynamics by laser speckle contrast imaging," Proc. SPIE 10685, Biophotonics: Photonic Solutions for Better Health Care VI, 1068509 (17 May 2018); doi: 10.1117/12.2306631

SPIE. Event: SPIE Photonics Europe, 2018, Strasbourg, France 


\title{
Investigation of speckle pattern dynamics by laser speckle contrast imaging
}

\author{
Anton Sdobnov*a, Alexander Bykov ${ }^{\mathrm{a}}$, Alexey Popov ${ }^{\mathrm{a}}$, Evgeny Zherebtsov ${ }^{\mathrm{b}}$, Igor MEglinski ${ }^{\mathrm{a}}$ \\ ${ }^{a}$ Faculty of Information Technology and Electrical Engineering, University of Oulu, Oulu 90570, \\ Finland; \\ ${ }^{\mathrm{b}}$ Aston Institute of Photonic Technologies, School of Engineering and Applied Science, Aston \\ University, Aston Triangle, Birmingham, UK B4 7ET
}

\begin{abstract}
Laser speckle contrast imaging (LSCI) is a simple and quite powerful method for visualization of flow, microcirculation and perfusion. In current study the speckle contrast variations towards breaking ergodicity conditions are considered with a final aim of envision a practical approach allowing real-time imaging of variations in dynamic properties of complex fluids with an opportunity of quantitative interpretation of the obtained flowing map. As example of systems with static to dynamic transition, melting of Intralipid samples were studied. Also, investigation of influence of static layer thickness above the dynamic sample on the ergodicity condition has been studied.
\end{abstract}

Keywords: Speckle contrast, speckle pattern, motion estimation, optical imaging, ergodicity

\section{INTRODUCTION}

Laser Speckle Contrast Imaging (LSCI), firstly introduced in the early 1980s [1], is a powerful low cost method allowing noncontact, full-field and real-time flow system mapping. It is widely known that when a diffuse object is illuminated with laser source a high-contrast speckle pattern is produced. Laser speckle is a random phenomenon which has been statistically described by Goodman [2]. The full theoretical background would be out of place in this paper. So, only the most important conclusions are given. The speckle contrast parameter is defined as:

$$
K=\frac{\sigma}{\langle I\rangle}
$$

where $\mathrm{K}$ is the speckle contrast, $\sigma$ is the intensity standard deviation and $<\mathrm{I}>$ is the mean intensity.

If any parts of illuminated object are moving it is introduces temporal fluctuations in single speckle intensity. These fluctuations are manifested in blurring of the speckle pattern during observation with fixed camera exposure duration. The blurring leads to a reduction in speckle contrast and can be used to obtain information about movement in object by statistical analyzing of blurring degree. The higher movements in object, the higher blurring degree. Therefore, changes in motion produces corresponding changes in speckle contrast value.

The firstly introduced and most commonly used method for LSCI measurements is the spatial statistics analyzing using sliding window over raw image [3]. Window of $5 \times 5$ or $7 \times 7$ pixel size is usually used to achieve good statistics but such kind of processing has disadvantage of speckle contrast image resolution reduction. To avoid this problem method of temporal statistics analyzing has been introduced [4].

So, various LSCI system setups has been successfully implemented for different applications such as assessment of influence of allergens on skin blood microcirculation [5], cerebral blood flow monitoring [6], clinical use on skin [7], monitoring of diabetic foot ulcers [8], in vivo characterization of tumor and tumor vascular network [9], etc. Also, LSCI potentially can be a powerful tool for monitoring of various processes in liquids. Some LSCI applications have been used for the studies of speckle patterns for Brownian motion [10,11] and turbulence [12].

Nevertheless, one important limitation of LSCI associated with non-ergodicity effect can dramatically affect results

*anton.sdobnov@oulu.fi; phone: +358449480406

Biophotonics: Photonic Solutions for Better Health Care VI, edited by Jürgen Popp,

Valery V. Tuchin, Francesco Saverio Pavone, Proc. of SPIE Vol. 10685, 1068509

(C) 2018 SPIE · CCC code: 0277-786X/18/\$18 · doi: 10.1117/12.2306631 
obtained by this technique. In the clinical experiments, sample usually heterogeneous and composed of both static and dynamic areas, or dynamic areas overlaid by static layers. Averaging ensemble is not equivalent for time and space. In practical case, duration of a single measurement which is equivalent to camera exposure time is much longer than relaxation time of moving contribution but much smaller than of the static contribution. So, the presence of static areas is manifested in non-ergodic speckle fluctuations, which lead to wrong data interpretation due the difference between spatial and temporal processing. It was shown that this difference can be used for more accurate speckle contrast imaging. [13] The introduced method, also allows the quantification of the eligibility conditions when the spatial approach can be replaced with the temporal approach for the obtaining of the images with higher resolution.

For this purposes the coefficient of speckle dynamics (CSD) has been introduced:

$$
C S D=\frac{2 K_{t}}{K_{s}+K_{t}},
$$

where $\mathrm{K}_{\mathrm{S}}$ is the speckle contrast over spatial window and $\mathrm{Kt}$ is the speckle contrast over temporal window. The higher values of CSD corresponds to the dynamic system while the lower values denoting the static areas.

In this study, with the main aim to assess limitations in the quantitative interpretation of speckle contrast images, the samples with various static to dynamic components ratio has been investigated. Melting of frozen Intralipid suspension has been observed as the process with transition from fully static to fully dynamic system. Also, liquid Intralipid covered by static layers with different thickness has been investigated.

\section{MATERIALS AND METHODS}

The following LSCI-based experimental setup illustrated on the Figure 1(a) has been used in this study: $13 \mathrm{~mW}$ laser diode working at $655 \mathrm{~nm}$ (Roithner LaserTechnik $\mathrm{GmbH}$, Austria) has been expanded by ground glass diffuser (Thorlabs, USA). CMOS- camera (Pixelink, Canada) with $1280 \times 1024$ pixels and $6.7 \mu \mathrm{m}$ pixel size was used in combination with $12 \mathrm{~mm}$ Kenko objective(Kenko Tokina Co., Ltd., Japan) for raw speckle image acquisition. Images obtained by camera were processed by custom made software in offline regime. For each speckle contrast image raw images from camera were obtained with $18 \mathrm{~ms}$ exposure.
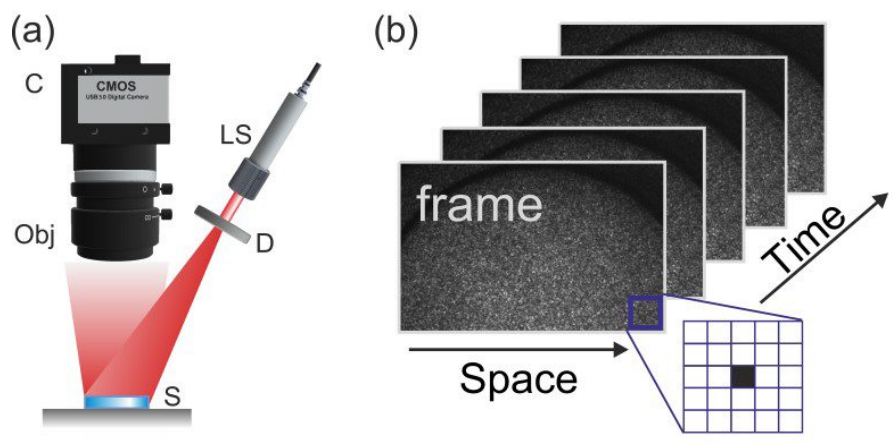

Figure 1. Schematic presentation of the laser speckle contrast imaging system (a). LS - laser source, D - diffusor, S sample, Obj - objective, C - camera. Spatial and temporal presentation of the obtained images (b).

In this study, $5 \%$ and $2 \%$ intralipid solution (Intralipid $\AA$, Fresenius Kabi, Sweden) was used for experiments. For the calculation of spatial and temporal speckle contrast 49 consecutive raw frames were used. Firstly, 49 frames were used to obtain one temporal speckle contrast image. Then, 49 spatial speckle contrast images has been calculated using 7x7 sliding window and averaged in final spatial speckle contrast image. Further, for the calculation of CSD map the obtained temporal and each of 49 spatial speckle contrast images has been used.

\section{RESULTS AND DISCUSSION}

To demonstrate possibility of monitoring processes with transition from fully static to fully dynamic state the further experiment has been prepared. $5 \mathrm{ml}$ Intralipid suspension was poured in Petri dish. Further, the sample has been placed 
into the fridge and was frozen at $-19^{\circ} \mathrm{C}$. Then, sample was placed on the damper table (Newport Inc., USA) and observed by LSCI system during the 3 hours at the room temperature $\left(21^{\circ} \mathrm{C}\right)$.

(a)
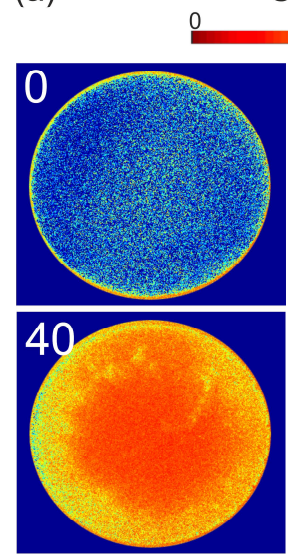

Speckle contrast, a.u.
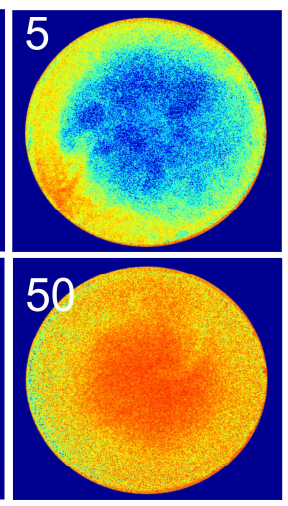

(b)

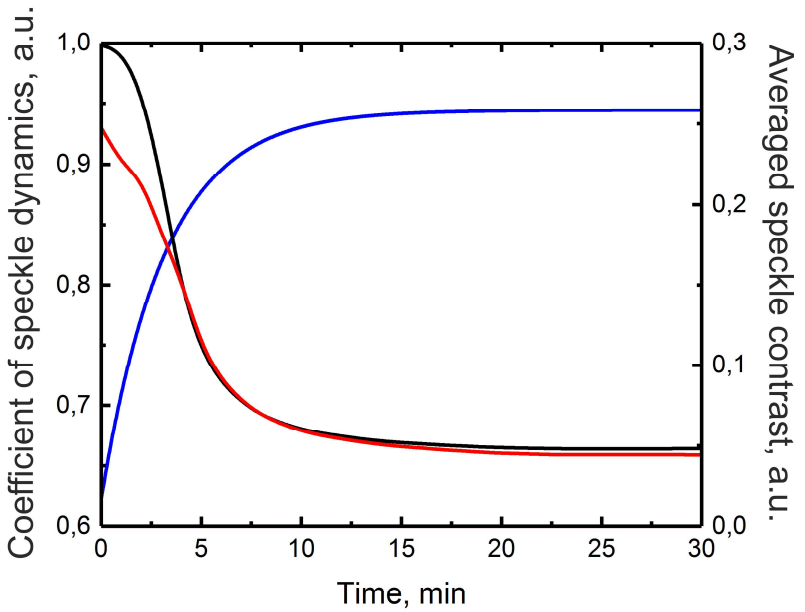

Figure 2. Temporal speckle contrast images of the Intralipid solution thawing process for 0, 5, 15, 40, 50 and 70 min after starting the experiment (a); Relation between averaged speckle contrast and time after starting the experiment: black and red lines represent, respectively, relative values of averagedspatial and temporal speckle contrasts in comparison with the averaged CSD values (blue line).

Figure 2(a) shows temporal speckle contrast images for 0, 5, 15, 4050 and 70 min after the beginning of melting process. In the beginning of melting speckle contrast takes the maximum values corresponding to static state. After 5 minutes from beginning of experiment speckle contrast values along the edges of Petri dish started to decrease which means that Intralipid sample is melting from the edges to the center. In 15 minutes the speckle contrast is significantly decreased over the whole sample area meaning that surface of the frozen Intralipid is covered by the thin dynamic layer.

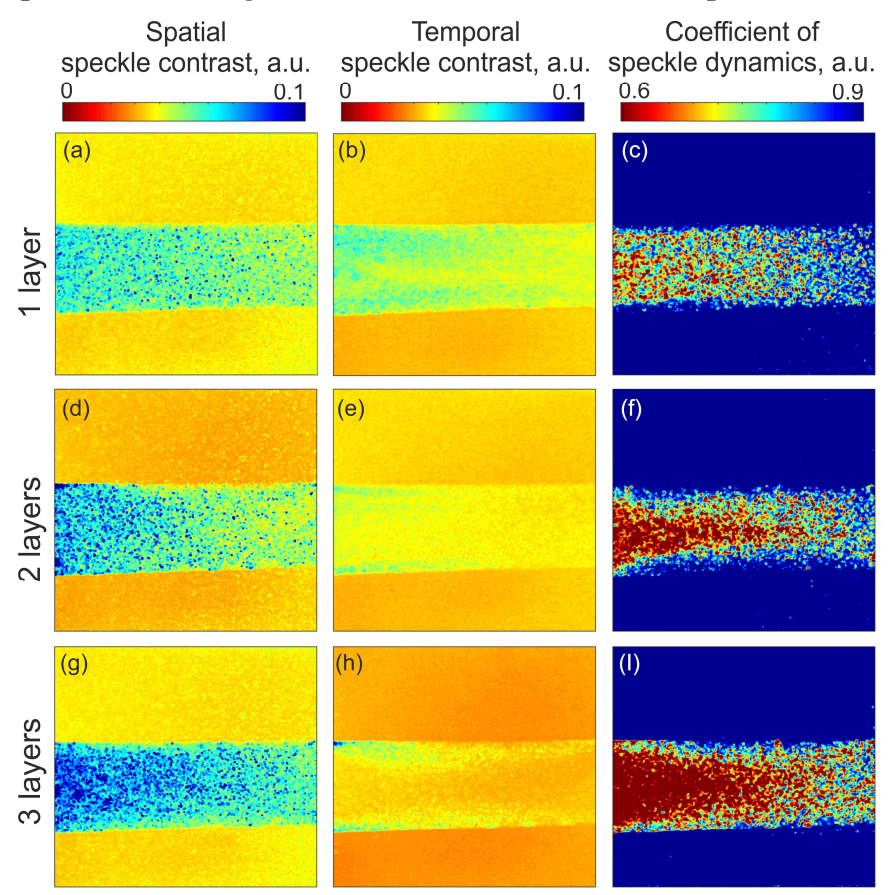

Figure 3. CSD maps of the Intralipid solution with 1 (c), 2 (f) and 3 (i) layers of tape and corresponding temporal (b, e and h) and spatial (a, d and g) speckle contrast images. 
Images for 40 and 50 minutes showed further melting of solid Intralipid sample under the liquid layer. After 70 min, the whole sample is completely melted and an increase of the speckle contrast values indicates that the speckles are defined only by Brownian motion.

Figure 2(b) shows the temporal and spatial speckle contrast values averaged over the sample area and averaged CSD values for the first 30 minutes of the melting process. It is clearly seen that in first 7 minutes spatial and temporal speckle contrast takes different values meaning presence of the static area. Also, after 7 min the CSD became saturated that corresponds to the dynamic state when the averaged speckle contrast values is equal for the temporal and spatial approach. This is means that in 7 minutes the static frozen area has been covered by the dynamic liquid layer of melted Intralipid. Moreover, the thickness of this layer is enough to consider the whole system as dynamic. So, this dynamic liquid layer can be considered as ergodic reference sample, described in Ref. [14,15].

To demonstrate influence of the thickness of static layer above dynamic one on the ergodicity conditions, the further experiment has been prepared. $2 \%$ Intralipid suspension has been poured into the plastic container. Then, 1, 2 and 3 layers of the tape (53 $\pm 2 \mathrm{mkm}$ thickness of 1 layer) has been placed on the suspension surface. For each experiment spatial speckle contrast, temporal speckle contrast and CSD images has been calculated. It is clearly seen that coefficient of speckle dynamics takes lower values for the experiment with 3 layers of tape due the fact that thickness of static area here bigger than in experiment with 1 and 2 layers of tape. So, presence of 3 tape layers correspond to fully non-ergodic state, while 2 and 1 layer corresponds to partially ergodic state. Thus, CSD mapping can be useful marker for changes in thickness of static layer embedded in dynamic one. For more advanced LSCI imaging, the proposed method can be used combined with optical clearing [16-18] allowing to reduce scattering of the static layer. Moreover, the approach, described above, can complement conventional speckle imaging approach in paint drying monitoring and/or roughness measurements [19].

\section{CONCLUSIONS}

We examined CSD mapping and LSCI approach at the conditions of static to dynamic state transition. CSD is a simple phenomenological approach which allows to assess quantitatively fraction of the dynamic and static parts of scattering medium. We showed that presence of dynamic layer above the static part of the sample allows to neglect all limitations of temporal processing of laser speckle contrast images. This result is in an excellent agreement with the results of alternative studies performed early. Also, we showed that proposed method can be used for assessment of influence of static layer thickness on the ergodicity of observed sample. Presented results have a strong potential to be used in clinic and industry.

\section{ACKNOWLEDGEMENTS}

This work was supported by the CIMO Fellowships program (21.3.16/TM-16-10089/CIMO Fellowship/WS19), EDUFI Fellowship program (13.10.17/TM-17-10655/EDUFI Fellowship/WS 19) and the Academy of Finland (Grant No. 290596),

\section{REFERENCES}

[1] Fercher, A.F. and Briers, J.D., "Flow visualization by means of single-exposure speckle photography," Opt. commun., 37(5), 326-330 (1981).

[2] Goodman, J.W., [Laser speckle and related phenomena], Springer Berlin Heidelberg, 9-75 (1975).

[3] Zakharov, P., Scheffold, F. and Weber, B. "Laser Speckle Imaging of Cerebral Blood Flow," Opt. Im. of Neocort. Dynam., 85 (2014).

[4] Cheng, H., Luo, Q., Zeng, S., Chen, S., Cen, J. and Gong, H., "Modified laser speckle imaging method with improved spatial resolution," J. Biomed. Opt. 8(3), 559-564 (2003).

[5] Kalchenko, V., Kuznetsov, Y.L., Prise, D., Meglinski, I. and Harmelin, A., "Mouse ear swelling test for weak allergens by using laser speckle imaging with the long exposure time," J. Biomed. Opt. 19(6), 60502 (2014).

[6] Abdurashitov, A. S., Lychagov, V. V., Sindeeva, O. A., Semyachkina-Glushkovskaya, O. V., and Tuchin, V. V., "Histogram analysis of laser speckle contrast image for cerebral blood flow monitoring," Front. Optoel. 8(2), 187-194 (2015). 
[7] Lee, K., Tchvialeva, L., Markhvida, I., Zeng, H., Doronin, A., Meglinski, I. and McLean, D.I. "Polarization Speckles and Skin Applications", Imag. Derm. 8 (2016) .

[8] Jayanthy, A. K., Sujatha, N., Ramasubba Reddy, M. and Narayanamoorthy, V. B., "Non invasive blood flow assessment in diabetic foot ulcer using laser speckle contrast imaging technique", SPIE BiOs, 89521D (2015).

[9] Kalchenko, V., Madar, N., Meglinski, I. and Harmelin, A., "In vivo characterization of tumour and tumour vascular network using a multi-mode imaging approach", J. Biophoton. 4(9), 645-649 (2011).

[10] Volpe, G., Volpe, G. and Gigan, S., "Brownian motion in a speckle light field: tunable anomalous diffusion and selective optical manipulation," Scientif. Rep. 4, 3936 (2014).

[11] Boas, D.A., Meglinski, I., Zemany, L., Campbell, L. E., Chance, B. and Yodh, A. G., "Diffusion of Temporal Field Correlation with Selected Applications," in CIS Selected Papers: Coh. Dom. Meth. Biomed. Opt. 2732 (1996).

[12] Meglinski, I.V., Boas, D.A., Campbell, L. E., Yodh, A. G. and Chance, B., "Diffuse photon probes as a tool for diagnostic of laminar and turbulent flow localized in highly scattering inhomogeneous media," Ann. Retr. Dep. Biochem. Biophys. (1995).

[13] Sdobnov, A., Bykov, A., Molodij, G., Kalchenko, V., Jarvinen, T., Popov, A., Kordas, K. and Meglinski, I., "Speckle dynamics under ergodicity breaking," J. Phys. D: Appl. Phys. 51(15), 155401 (2018).

[14] Medebach, M., Freiberger, N. and Glatter, O., "Dynamic light scattering in turbid nonergodic media." Rev. Sci. Instr. 79, 073907 (2008).

[15] Haro-Pérez, C., Ojeda-Mendoza, G.J. and Rojas-Ochoa, L.F., "Three dimensional cross-correlation dynamic light scattering by non-ergodic turbid media." J. Chem. Phys. 134, 244902 (2011).

[16] Sdobnov, A. Y., Darvin, M. E., Genina, E. A., Bashkatov, A. N., Lademann, J. and Tuchin, V. V., "Recent progress in tissue optical clearing for spectroscopic application," Spectrochim. Act. A: Molec. and Biomolec. Spectrosc. 197, 216-229 (2018).

[17] Sdobnov, A. Y., Tuchin, V.V, Lademann, J., and Darvin, M.E., "Confocal Raman microscopy supported by optical clearing treatment of the skin - influence on collagen hydration," J. Phys. D: Appl. Phys. 50 (28), $285401(2017)$

[18] Sdobnov, A. Y., Darvin, M. E., Lademann, J. and Tuchin, V. V., “A comparative study of ex vivo skin optical clearing using two-photon microscopy," J. Biophotonics, 10(9), 1115-1123 (2017).

[19] Arizaga, R., Grumel, E.E., Cap, N., Trivi, M., Amalvy, J.I., Yepes, B. and Ricaurte, G., "Following the drying of spray paints using space and time contrast of dynamic speckle," JCT research, 3(4), 295-299 (2006). 